This document is published in:

Murgante, B. et al. (eds.) (2011) Computational Science and Its Applications - ICCSA 2011: International Conference, Santander, Spain, June 20-23, 2011. Proceedings, Part IV. (Lecture Notes in Computer Science, 6785). Springer, 488-501. DOI: http://dx.doi.org/10.1007/978-3-642-21898-9_41

(c) 2011 Springer-Verlag Berlin Heidelberg 


\title{
Towards Combining Individual and Collaborative Work Spaces under a Unified E-Portfolio
}

\author{
Hugo A. Parada G., Abelardo Pardo, and Carlos Delgado Kloos \\ Department of Telematic Engineering, University Carlos III of Madrid, Spain \\ \{hparada, abel, cdk\}@it.uc3m.es http://gradient.it.uc3m.es
}

\begin{abstract}
E-portfolios in learning environments have been attributed numerous benefits and their presence has been steadily increasing. And so has the variety of environments in which a student participates. Col-laborative learning requires communication and resource sharing among team members. Students may participate in multiple teams throughout a long period of time, sometimes even simultaneously. Conventional e-portfolios are oriented toward showcasing individual achievements, but they need to also equally reflect collaborative achievements. The ap-proach described in this paper has the objective of offering students an e-portfolio as a local folder their personal computer containing a com-bined view of their individual and collaborative work spaces. The content of this folder can be synchronized with a remote server thus achieving resource sharing and publication of a clearly identified set of resources.
\end{abstract}

Keywords: e-portfolio, collaborative learning, personal learning environment.

\section{Introduction}

Although the presence of information and communication technology in the realm of learning experiences has been increasing significantly over the years, an equally significant increase has also been perceived in the orchestration of any experience. Scenarios in which students were simply given a set of resources with some basic (and typically fixed) organization have evolved to learning scenarios in which students interact in groups, search for additional resources, communicate using different structures and patterns, etc.

This emerging scenario requires highly flexible learning spaces to provide the right environment for each stage of a learning experience. Learning Management Systems (LMS) were initially conceived to solve administrative issues and their structure relies heavily in the concept of "course" or "classroom". But this approach is now showing clear signs of fatigue. There are several forces that are re-shaping the landscape. Two of them are relevant to the approach presented in this document. The first one is that learning is relying more in multi-modal 
communication. The exchange of information between students and instructors or among students themselves supported by different tools and technologies, is shown to have a positive effect in the learning process. Collaborative learning, for example, proposes the creation of students groups in which the interaction among team members is the basis for achieving a set of objectives.

The second changing force is "student-centered learning". The student is now the central part of the strategy to achieve a successful learning experience. Activities now may require students to individually collect, organize and create new resources as a consequence of the learning process.

When combining these two forces, the need for flexible learning spaces emerges, and this need is not fully covered by conventional LMSs. Tools such as for example e-portfolios or social learning platforms are emerging as solutions to accommodate this type of scenarios. A student may participate in numerous groups. Some of them may be embedded within a course, and others may step out of the course boundaries. From the point of view of the instructors, an efficient and flexible group management would help cope with the increase of complexity when orchestrating non-trivial collaborative experiences.

In this document a platform is proposed to offer students a folder in the space of their personal computer with a structure to combine a personal area, a public area that is sent to a remote server and available in the net, and as many shared spaces as the number of groups in which they participate. The work focuses on the resource management aspect and how to manage efficiently a large number of student groups.

\section{Related Work}

Portfolios have been used for years in different disciplines to support personal development. Due to the adoption of information and communications technologies to implement it, they were called E-portfolios; which were initially defined as a selective and structured collection of digital resources gathered for the purpose of documenting experiences, storing intellectual assets, showcasing achievements, assessing personnel, and retrievable using a medium such as the Web, DVDs, etc $[3,8]$. But such an ample definition, when used in the context of a learning experience, has given rise to an equally large set of potential benefits. A review of the research literature shows that e-portfolios promote more effective learning, can be used as a form of assessment, may facilitate employment, encourage life-long learning, allow reflection on the artifacts included, obtain feedback from peers, and more generally, allow a different mode of interaction.

But as some authors have pointed out $[2,7]$, much effort has been devoted to describe and emphasize the advantages for institutions, instructors or "learning" in general, but not so much to the impact on the students.

In [12] Jafari describes the e-portfolio role in higher education and points out the danger derived from the variety of meanings that the term represents for different actors of the educational arena. Educators, career center directors, department chairs and students all have different views of what an e-portfolio 
is and how it should be used. This variety of views has caused confusion and hindered the adoption of e-portfolios. The proposed solution is the use of different identifiers preceding the term "e-portfolio" to help clarify its functionality. If this recommendation were to be used in this work, the proposed functionality could be called "local folder-based e-portfolio".

In the education area e-portfolios have been used to follow the students progress as to how they are achieving the course goals; and to become aware of student performance during the course based on it. Highlighting this line, in [4] Bhattacharya et. al. propose an e-portfolio aimed at raising awareness of the set of skills acquired by the students by selecting and analyzing the most significant resources. This task is complicated when students will have a mixture of resources created individually and collectively in various teams. A central repository combining this resources is the strategy proposed in this paper.

One important aspect of the use of e-portfolios in an educational environment is how easy knowledge from different sources can be integrated. In [5] the use of student e-portfolios is proposed in science and technology both for group and individual tasks. The authors claim that students working in groups experienced a richer environment when compared to those working individually. However, no technical details are given as to how the e-portfolio is implemented.

A critical stage when using e-portfolios in a learning environment is their deployment and adoption by the students. In [16], Murray and Currant point out that engagement by students is paramount for an e-portfolio to have an impact. Typical web-based platforms may encounter some resistance from the students due to their "rigidity". The approach proposed in this paper is based on managing a folder stored in the user local space (for example, a personal computer). On the other hand regarding e-portfolio adoption in [11] Hämäläinen et. al. consider the following two aspects as key: first, that the integration of information created during the course into an e-portfolio from different sources should be made as simple as possible; and secondly, the e-portfolio has to be wellorganized. Our proposal considers these two aspects by allowing the planning and creating of a flexible reference structure involving links to external folders by instructors in order to host the student's artifacts.

Collaborative learning is conceived as an effective strategy to improve learning, and it can be improved by using tools supported by pedagogical goals. In [7], Carrol et. al. present a tool to encourage both reflective thinking and collaborative reflective learning. The work describes Dotfolio, a tool designed and used to support collaborative learning which includes functionality to encourage the exchange of ideas among teachers and students, collaborative reflection about course topics, etc. This tool highlights the importance of collaborative activities as the result of individual efforts rather than individual isolated activities. Our work proposes a solution oriented towards scenarios in which students participate in multiple group activities, some of them simultaneously, for a long period of time.

Most e-portfolio implementations used to be mainly oriented to sharing individual knowledge and experiences (after all, that was the essence of the classic 
portfolio). However this trend is changing. In [14] Limanauskiene et. al. claim that the success of e-learning depends not only on the individual's abilities but also on how individuals share these abilities with others for building innovations. Based on this premise they propose a group e-portfolio implementation aimed at fostering the sharing of knowledge among learning community members. This work proposes the creation of a shared space for individual and collaborative tools so that learners can have a group environment from which individual eportfolios can be accessed. The group portfolio is conceived as a portal integrating environments such as social networks, Learning Management Systems, learning community portals and personal portfolios. However, the strategy adopted is based on users sharing their individual portfolios within a group. Our contribution aims at maintaining a clear yet intuitive distinction between different collaborative spaces and the individual portfolio.

Our work is similar in spirit to that presented in [10]. The process of design an e-portfolio tool is described in which collaborative learning has a paramount presence. In [19], Wang describes a collaborative learning experience in which students use the cloud application "DriveHQ" to share all the documents within the teams.

The work presented in this paper is similar in the sense that it extends the eportfolio functionality by combining the student individual work space (his/her personal computer) with the various collaborative environments in which he/she takes part for an arbitrarily long learning experience. The focus of the contribution is in offering a single folder to be used in a personal computer in which students may instinctively work on private documents, contribute resources to a group activity, or select a subset of this resources to make them publicly available on the net. The proposed approach can be easily complemented with current platforms to offer virtual spaces to interact containing other conventional tools such as forums, chats, etc.

The revision of the literature on e-portfolios, shows that most works propose mechanisms to support the individual work of the students. So far they do not go into great detail as to an e-portfolio contribute to collaborative work; and how they can be applied to a specific scenario. It is also evident that aspects related to how to combine individual e-portfolio with results derived from collaborative were not addressed.

Taking the advantages of individual e-portfolio as starting point in this work we extend its functionality by integrating it with resulting artifacts from group work. It is based on a "reference work space" which allows designing an initial structure of both the individual and shared work space. This reference work space contains the combination of individual and groups spaces and can then be easily replicated to be used by the students in a course. In addition our proposal facilitate planning the course activities, monitoring student progress, and make changes to the work space structure when new requirements arise. 


\section{E-Portfolio for Multi-collaborative Environments}

As described in Section 2, e-portfolios are typically used as a main tool to support the personal learning process and showcase individual student achievements. By preparing these resources and sharing them with peers, students may also work on reflection and obtain valuable feedback. The work proposed in this paper tries to embrace these advantages and combine them with a collaborative setting. In order to simplify its presentation, a specific scenario has been created. Let us consider a one semester course which is part of an engineering degree program. The course adopts a collaborative team strategy for a software development project. The objective is to expose students to professional practices. Several instructors are assigned to different sections of the course. During the semester students must take part in both individual and group activities. Each activity is related to a project with the objective of producing an artifact. These artifacts are stored in a work space which is organized into several folders.

The artifacts may be of two types: those generated as results of the individual work, and those derived from team work. Therefore the student work space is divided into two areas: personal and team work spaces. Students that are members of the same team have access to the same content in a folder. Individual spaces remain private. Students may submit new artifacts at any time to a central server. For those submissions, they choose to place them on the private or team work areas. The type of feedback received from the tutors on the artifacts may also be individual or from the team members. Teams are created based on the instructor's observation during the first weeks of the course and work is carried out independently for each team. Team structures may vary slightly during the semester depending on unforeseen circumstances (for example, severe conflicts). At any time, each student has an area where the individual as well as team artifacts are stored.

This environment offers a more flexible space for students to manage the information available during the course so that they can make a more productive use of it. Additionally both students and teams can host additional material and links to external information sources in their work space; and a set of artifacts in a specific area can also be distinguished as openly available through a web page. With this approach, we want the students to make a more efficient use of the course resources, and for tutors to simplify the management of team management and supervision. Figure 1 illustrates the scenario we have just described.

But managing this multi-collaborative scenario translates into a set of tasks for the instructors: define the initial structure of these work spaces, decide team sizes, folder structure, access policy, permissions, initial project documentation, etc. Ideally, the combination of individual and group spaces should be created for each student. The proposed tool provides support to lower the administrative tasks to manage a set of students which are given access to an individual folder in which they can produce any artifact, plus a team space in which these artifacts are created collaboratively. The system offers primitive operations to define access policies. Based on these primitives the instructors can describe the team definition rules by assigning the users to each work space. During the course, 


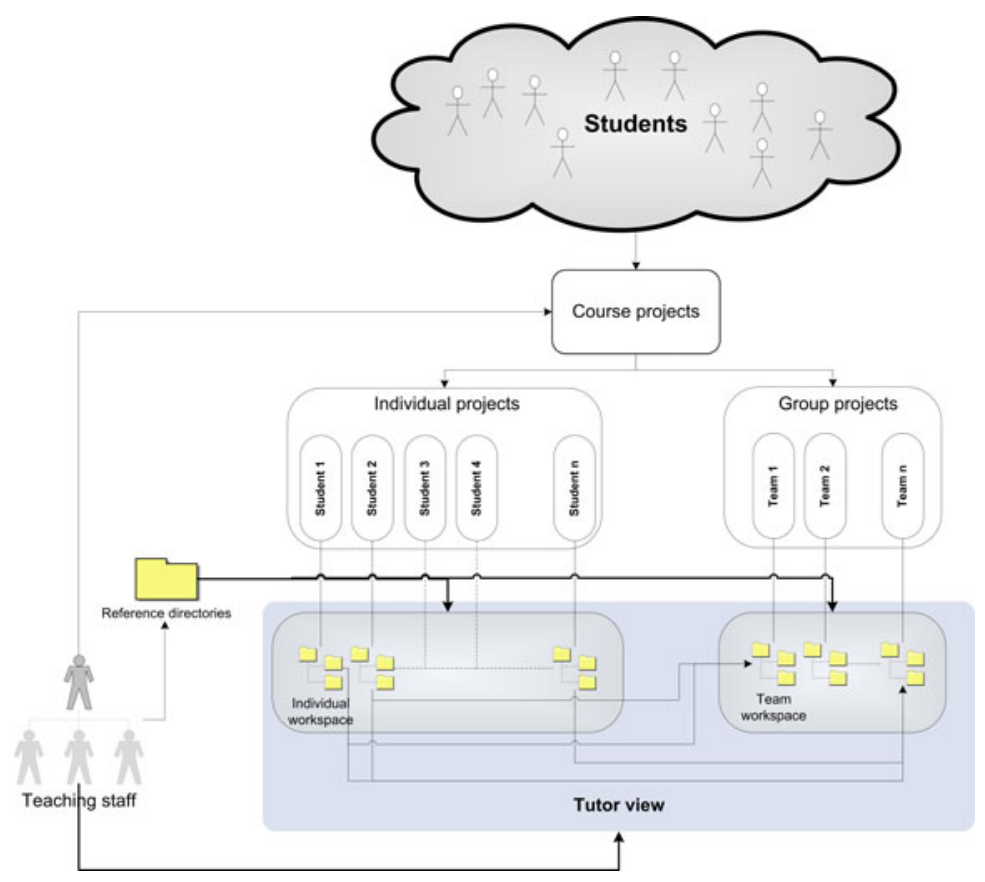

Fig. 1. E-portfolio scenario

tutors can interact with student either by reading, modifying and adding new information to the content folders or by creating new folders. This interaction can occur at any time during the course or can be regulated through the definition of a set of timestamps defining a window during which operations are allowed in a folder. This mechanism is used to enforce submission deadlines. Tutors may also create additional teams during the course enactment.

In order to manage the complexity derived from this scenario we propose an e-portfolio that is managed directly by the student just like any other regular folder in his/her personal computer. This e-portfolio expands from the individual space to a multi-space. It can be created with an initial basic structure. Tutors can modify the initial structure during the course and these changes are then transferred to the student spaces. Tutors can create different views of these spaces by selecting just the relevant parts at each moment. Within a single space, tutors may compare all the resources that a student is using in the different scenarios. Analogously, students are offered a folder in their individual computers containing all the resources in which they worked for the different scenarios.

The process of building and managing these new folders is structured into the following steps: design the initial structure of the portfolio, assign students to the teams, and create the e-portfolio. First, instructors define the basic elements from which the student spaces will be instantiated. Then a set of templates that represent folders and sub-folders are defined. We have called these templates the 
reference directories. After that the links pointing to the collaborative folder are also defined.

Once these spaces are defined, students are then assigned to the teams. For each grouping, the instructor defines a set of rules to replicate the team's shared spaces withing the individual spaces. All course students will have assigned a directory reference where both the individual and team sources are available.

Once the folder structure and the group rules are created, the reference directories with the initial content are replicated. Each student starts with the same initial local folder with the same structure with as many links to the team shared spaces are required.

\section{Proposed Implementation}

In the specific context of engineering studies, some recent studies suggest [1] that team projects are important components of undergraduate curricula to expose students to professional practices. The same tendency is observed in the context of Internet where numerous web-based portals for software projects have appeared (see [6] for a survey). Version control systems (henceforth simply VCS) are tools used in these scenarios to exchange all types of resources among team members and maintain the history of changes. There are numerous VCS tools available and their functionality has been increasing steadily over the years; among the more popular we highlight Subversion [9], CVS ${ }^{1}, \mathrm{Mercurial}^{2}$, and recently $\mathrm{Git}^{3}$.

Although our proposed digital portfolio structure could be implemented using any VCS, we have selected Subversion because it provides a more fine-granularity access control policy. Thus our approach allows a group of users to share a set of directories stored in a centralized server. With the current approach, the use of the digital portfolio has the added value of exposing students to the use of these industry-type tools typically used in team based projects.

In educational institutions, however, these systems are typically introduced (if at all) in the last courses of a program. But as stated by Reid [18], there is clearly a pedagogic value in using them also in the early courses. Other authors even consider the use of these tools "imperative" [15]. In the portfolio application, though, the objective is allow the creation of as many shared and individual spaces as needed for a large set of students.

The implementation of the proposed digital portfolio is based on three data representations: a set of reference work spaces, a set of connecting rules, and a set of group definitions.

\subsection{Reference Work Spaces}

The first concept on which this application is based is the so called "reference work space". It is simply a folder containing the initial structure of a work space

${ }^{1}$ www.cvshome.org

${ }^{2}$ mercurial.selenic.com

${ }^{3}$ git-scm.com 
to be offered to a set of students. The application allows the manipulation of a set of these reference work spaces to be later cloned as many times as specified by the group definitions.

The most important feature of these work spaces is that they are replicated into multiple instances while maintaining the relation with the initial structure. This relation, together with the capability that VCS allow to "merge" changes introduced by the instructors on the references for a fast propagation of these changes to the replicated instances.

The change propagation is specially useful when, half way down the life of a shared space, an initial resource (copied in all instances) needs to be modified, or the folder needs to be internally reorganized. Instructors may apply these changes to the reference work space and the tool propagates them to the different student spaces. The process is automatic as long as the underlying VCS is capable of merging these changes. When this is not possible, there are mechanisms to solve these conflicts, although they require the intervention of the instructors.

The idea behind maintaining several reference work spaces is to create one for each different "realm" where students are supposed to work. For example, a reference work space would define the initial structure of the individual portfolio, whereas each group in which the student participates would have its corresponding reference space as well.

\subsection{Connecting Rules}

But typically, students participate in an ever increasing variety of communities. Sometimes these communities are within one single course, some others span across course boundaries. In order to offer the combined view of all their work spaces, a set of rules needs to be defined such that all the student spaces are connected to offer an initial identical folder.

The notion of "connecting" two folders is a well known technique used internally by operating systems to simplify file management. The terms usually used are "symbolic links", "hard links" or "shortcuts". The idea is quite simple. At an arbitrary point in a file hierarchy a different folder is attached to appear as if it is physically at that point.

The proposed approach follows this analogy to allow the definition of a set of rules to combine a subset of the reference work spaces defined in Section 4.1. Figure 2 shows an example of the configuration files containing these two ingredients. In the left side of the figure three reference work spaces (with names "tree1", "tree2", and "tree3") are defined pointing to three locations in the central repository. In the left side, two connecting rules are defined. The first one connects the space in "tree2" as an additional child at the top level of "tree1". Analogously, the second rule defines the space in "tree3" also as another child at the top level of "tree1". As a consequence of these definitions a single individual work space with the structure of "tree1" is defined that has as two sub folder in the top level the copy of the structures "tree2" and "tree3" respectively.

This resulting folder is then cloned using the group definitions explained in the following section. 


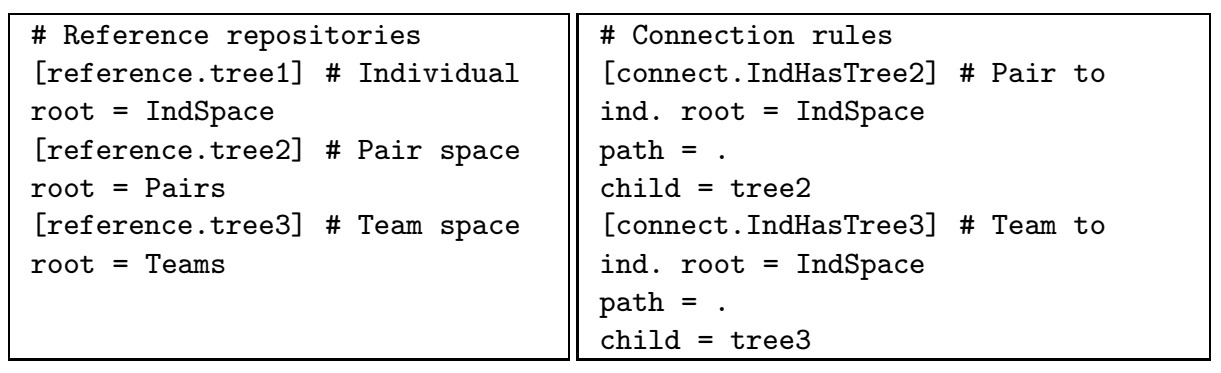

Fig. 2. Reference Repositories and Connecting Rules Configuration

\subsection{Group Definitions}

In a generic learning scenario, a set of users may be organized into a set of groups with different arrangements depending on multiple variables. For example, within the same course, activities can be carried out in teams or in groups formed by one representative of each team. If we widen the scope, students may be grouped differently in several courses of the same degree program.

The discussed approach assumes a global user community in which at least two roles are defined: students and staff. Students can be divided following several grouping policies. Each grouping policy is captured with the following data:

- Name of the grouping policy to be used in grouping commands.

- Reference to one of the reference repositories (as described in Section4.1).

- A prefix to be used to generate the names of the instances derived from the policy.

- The number of digits to use to create the group number to append to the previous prefix.

- A space separated list of staff users that may access the instances of the group spaces.

- A comma separated lists of groups. Each group is a space separated list of strings in which the first one is the group name, and the following are the user names of the group members.

Figure 3 shows the definition of three grouping policies. The "group.Individual" policy states that the reference work space "tree1" should be cloned six times, and each of them should be accessed by a single user. Also, the prefix policy is defined such that the user name is used as the name for the work space. Furthermore, the access to these work spaces is allow to all three staff members.

The second grouping policy (with name "group.Pairs") states that the "tree2" reference repository should be replicated for the three pairs of students described. These work spaces will be named with the prefix "Pair_" followed by correlative numeric values represented by two digits. The access to these work spaces is allowed only to two of the three instructors.

The third and last grouping policy (with name "group.Triplets") states that two team instances should be created with names starting with the prefix "Team_" 


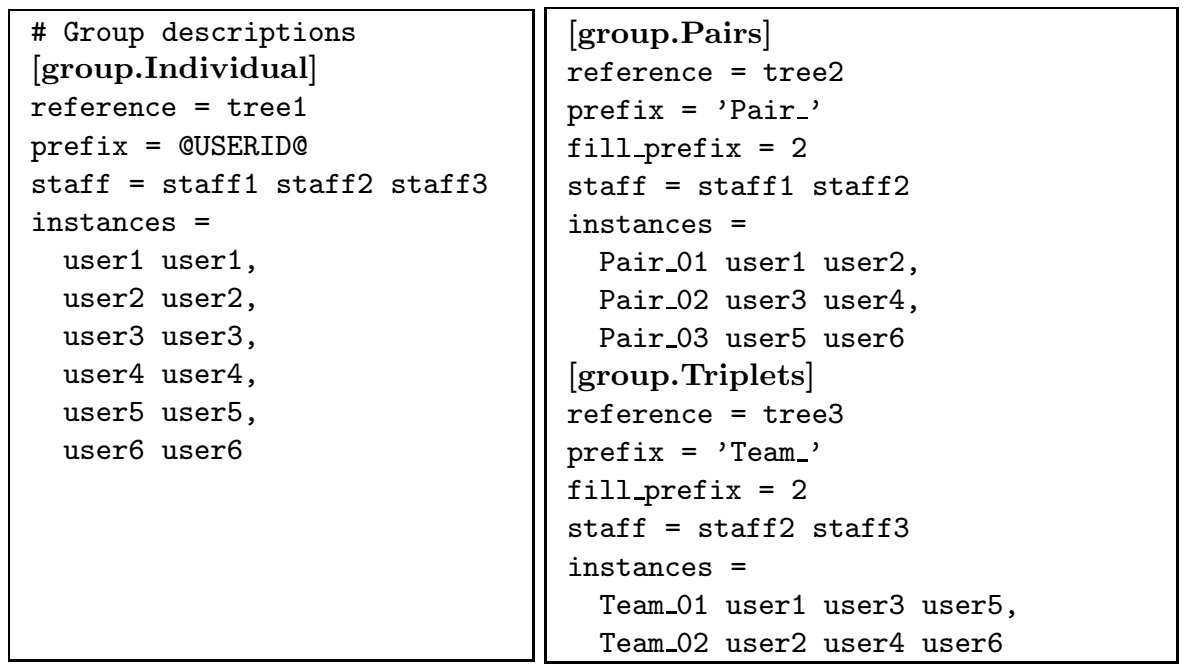

Fig. 3. Group Descriptions for individual, pairs and team cloning

followed by a two digit number. Two other instructors are allowed to access these work spaces.

\subsection{Portfolio Creation and Manipulation}

For the sake of clarity, an example of how the proposed approach can be deployed is described in detail in this section. Let us assume that three instructors inst1, inst2, and inst3, are in charge of creating, deploying and supervising the work of a set of six students (with user names student1, through student6).

For this user community, three working spaces are considered:

Individual. Each student has an individual work space with an initial structure.

Such space contains a clearly identified folder the content of which will be available in the net. All instructors have access to this area.

Pair Group. Students are grouped in pairs (three pairs in the course) to carry out a set of activities. There is one work space for each pair of students shared among the pair members. Each instructor supervises on of the pairs.

Teams. Students are also grouped in teams of three (two teams in the course) to carry out a second set of activities. There is one work space shared among the team members. Instructors inst 1 and inst 2 supervise the first team, and inst 2 and inst3 supervise the second team.

Figure 4 shows the three reference work spaces initially created by the instructors. Only the folders are shown to simplify the view. Instructors place in these areas all the initial files to be given to the students. For example, folder public in the tree1 may include a file stating that the folder is meant to contain documents to publish in the net. Thus, any document uploaded in that area becomes public once it is committed to the central repository. 

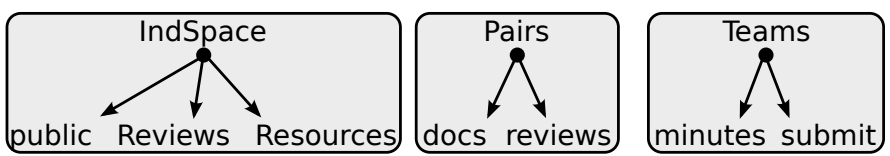

Fig. 4. Three reference work spaces created by the instructors

The definition of these three directories as reference work spaces is accomplished with the configuration shown in the left side of Figure 2. Next, a monolithic work space is created by defining how the three work spaces are combined. In this case, we want that every instance of the individual folder contains two instances of the pairs and team work spaces at the top level. This accomplished with the configuration rules shown in the right side of Figure 2.

Finally, the way in which reference spaces are to be replicated is captured by the configuration shown in Figure 3. The individual work space is replicated for each student, and at the same time combined with a space shared by the pairs and another space to be shared by the teams. Additionally, the right permissions are defined for each folder in the space.

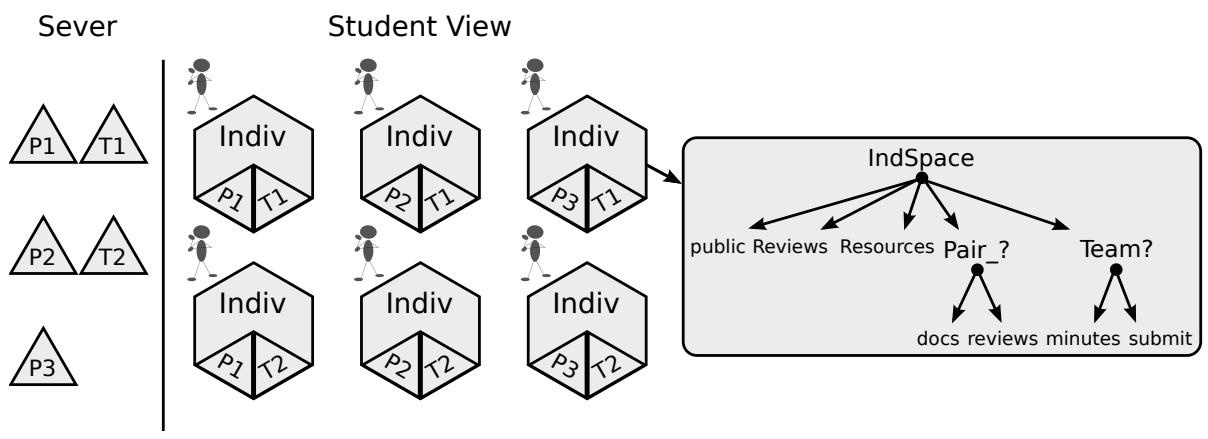

Fig. 5. Final set of work spaces in server and student view

Figure 5 shows the resulting global working environment once the configuration rules are interpreted and executed. Six individual spaces are created by cloning the reference, and their corresponding working spaces for the work in pairs and teams appear as another regular folder.

\section{Discussion and Future Work}

With the proposed approach, several important features are combined into a single solution. In its simplest form, instructors may create an initial structure of a folder where students collect the material related to the learning experiences in 
which they are participating. This initial structure contains a public area which is publicly available to showcase the achievements. Instead of designing a page in a web-base platform, the work in their regular documents and the work in a page can be intermixed.

When students become members of a team as part of a collaborative experience, their personal space is modified with the appearance of a folder shared among all team members. Access rights for the documents is given only to the team members and staff. The exchange of documents within the group and with the tutor is significantly simplified. These documents are sharing a space which is stored remotely. When, either within the same course, or an entirely different experience, students participate in other groups, their personal space is again extended with a new folder. Access rights can be properly adjusted even to offer a window of validity during which changes are allowed in the contained resources.

A concrete example of a collaborative activity will help to clarify the proposed functionality. Let us assume a course with 20 students in which a "jigsaw" [13] pattern is used. Initially students are divided into four "expert profiles". There are five students in each profile. Initially all students have an initial folder with their private files. When divided into these four profiles, four groups are created. Each group is given a different set of resources to review and exchange their remarks. For each student, a new folder appears with the appropriate resources, and the folder is shared among the peers with identical profile.

In the second step, 4 member team work spaces are created taking one student from each profile. A second work space appears for each team in which a new set of resources is exchanged. At the same time, instructors are provided full access to these spaces to be able to monitor their progress as well as to provide feedback about the different tasks.

With the proposed solution, the creation/deletion of these new spaces can be done easily, for an arbitrarily large number of students, fully embedded into their personal learning environment, and with the possibility of easily applying changes to any of the resources.

A first set of pilot experiences were conducted in which students were given access to a set of work spaces not yet integrated under the proposed single folder view [17]. Students were given a first shared space in which a set of activities were carried out in pairs. At a second stage, with the same cohort, teams of four students were created, and a second shared space was created from an initial structure given by the instructors.

Students adopted the working paradigm with no problem. As a matter of act, the proposed scheme complemented nicely the "web disk service" offered by the university. Such disk can only be used for storing individual files, so the newly created spaces were ideal for exchanging documents within the teams.

The next step is to deploy a single unified view of the multiple work spaces. Together with this deployment, the course will include activities specifically oriented to the publication of certain resources in a place publicly available on the net. Once this deployment is finished, the more challenging task of assessing its impact in a learning experience will be tackled. 
Finally, our approach was designed to allow its integration with other platforms of the learning ecosystem. Hence these topics have been keep out of scope of this paper. Then, several open issues should be taking into account in order to integrate our solution to this "ecosystem"; specifically we consider face the following: providing an access point to the contents generated by students in collaborative an social platforms; definition of mechanism to allow integration with learning management systems.

\section{Acknowledgment}

Work partially funded by the Learn3 project, "Plan Nacional de I+D+I TIN200805163/TSI", the Consejo Social - Universidad Carlos III de Madrid, the Acción Integrada Ref. DE2009-0051, and the "Emadrid: Investigación y desarrollo de tecnologías para el e-learning en la Comunidad de Madrid" project (S2009/TIC-1650).

\section{References}

1. Association for Computing Machinery. Computer Science Curriculum 2008: An Interim Revision of CS 2001. IEEE Computer Society, Los Alamitos (2008)

2. Ayala, J.: Electronic Portfolios for Whom? Educause Quarterly 29(1) (2006)

3. Barrett, H.: ICT support for electronic portfolios and alternative assessment: The state of the art. In: World Conference on Computers and Education (WCCE), pp. 1-11 (2001)

4. Bhattacharya, M., Heinrich, E., Rayudu, R.: Work In Progress: E-portfolios in Computer Science and Engineering Education. In: Proceedings of the Frontiers in Education. 36th Annual Conference, pp. 11-12 (2006)

5. Bhattacharya, M., Novak, S.: An Integrative Model for the Evaluation of Eportfolios. In: International Conference on Advanced Learning Technologies, July 2007, pp. 215-216. IEEE Press, Los Alamitos (2007)

6. Cabot, J., Wilson, G.: Tools for Teams: A Survey of Web-Based Software Project Portals. Dr. Dobb's (October 2009), http://www.drdobbs.com/tools/220301068 (last accessed December 2010)

7. Carrol, N.L., Markauskaité, L., Calvo, R.A.: E-Portfolios for Developing Transferable Skills in a Freshman Engineering Course. IEEE Transactions on Education 50(4), 360-366 (2007)

8. Challis, D.: Towards the mature ePortfolio: Some implications for higher education. Canadian Journal of Learning and Technology 31(3) (2005)

9. Collins-Sussman, B., Fitzpatrick, B., Pilato, C.: Version control with subversion. O'Reilly Media, Cambridge (2004)

10. Gerbic, P., Maher, M.: Collaborative self-study supporting new technology: The Mahara e-portfolio project. In: Proceedings of ASCILITE Melbourne 2008, pp. 320-324 (2008)

11. Hämäläinen, H., Ikonen, J., Porras, J.: Developing Technical E-portfolio Construction Process. In: 2009 Ninth IEEE International Conference on Advanced Learning Technologies, July 2009, pp. 711-712 (2009)

12. Jafari, A.: The "Sticky" ePortfolio System: Tackling Challenges and Identifying Attributes. Educause Review 39(4) (2004) 
13. Johnson, D., Johnson, R.: Learning together and alone. Cooperative, competitive, and individualistic learning. Allyn and Bacon, 160 Gould Street, Needham Heights, MA 02194 (1994)

14. Limanauskiene, V., Blazauskas, T., Binkis, M.: Knowledge Sharing with Group Portfolio. In: International Conference on Advanced Learning Technologies, pp. 428-430 (July 2009)

15. Linder, S., Abbott, D., Fromberger, M.: An instructional scaffolding approach to teaching software design. Journal of Computing Sciences in Colleges 21(6), 238-250 (2006)

16. Murray, C., Currant, N.: E-portfolios along the Lifelong Learning Cycle: Differences between Use, Pedagogy and Context. In: International Conference on Advanced Learning Technologies, pp. 491-493. IEEE, Los Alamitos (2006)

17. Pardo, A., Delgado Kloos, C.: SubCollaboration: Large Scale Group Management in Collaborative Learning. Software: Practice and Experience 41(4), 339-465 (2011)

18. Reid, K., Wilson, G.: Learning by doing: introducing version control as a way to manage student assignments. In: Proceedings of the 36th SIGCSE technical symposium on Computer science education, pp. 274-276. ACM, St. Louis (2005)

19. Wang, Q.: Design and evaluation of a collaborative learning environment. Computers \& Education 53(4), 1138-1146 (2009) 